\title{
Genetic relationships among Enterococcus faecalis isolates from different sources as revealed by multilocus sequence typing
}

\author{
X. Chen, Y. Q. Song, H. Y. Xu, B. L. G. Menghe, H. P. Zhang, and Z. H. Sun ${ }^{1}$ \\ Key Laboratory of Dairy Biotechnology and Engineering, Ministry of Education, Inner Mongolia Agricultural University, Hohhot, 010018, \\ P. R. China
}

\begin{abstract}
Enterococcus faecalis is part of the natural gut flora of humans and other mammals; some isolates are also used in food production. So, it is important to evaluate the genetic diversity and phylogenetic relationships among E. faecalis isolates from different sources. Multilocus sequence typing protocol was used to compare 39 E. faecalis isolates from Chinese traditional food products (including dairy products, acidic gruel) and 4 published E. faecalis isolates from other sources including human-derived isolates employing 5 housekeeping genes (groEL, $\operatorname{clp} X, \operatorname{rec} A, \operatorname{rpoB}$, and $\operatorname{pep} C)$. A total of 23 unique sequence types were identified, which were grouped into 5 clonal complexes and 10 singletons. The value of standardized index of association of the alleles $\left(\mathrm{I}_{\mathrm{A}}{ }^{\mathrm{S}}=0.1465\right)$ and network structure indicated a high frequency of intraspecies recombination across these isolates. Enterococcus faecalis lineages also exhibited clearly source-clustered distributions. The isolates from dairy source were clustered together. However, the relationship between isolates from acidic gruel and one isolate from a human source was close. The MLST scheme presented in this study provides a sharable and continuously growing sequence database enabling global comparison of strains from different sources, and will further advance our understanding of the microbial ecology of this important species.
\end{abstract}

Key words: Enterococcus faecalis, multilocus sequence typing, genetic relationship

\section{INTRODUCTION}

Enterococcus faecalis is part of the natural gut flora of humans and other mammals and grows under restricted environmental conditions. In humans, they can be considered as opportunistic pathogens and may be present in vegetables, fruits, meat, and milk, as a consequence

Received March 14, 2015.

Accepted April 27, 2015.

${ }^{1}$ Corresponding author: sunzhihong78@163.com of fecal contamination. However, current legislation sets no limit for the presence of this species in food, because it is not always associated with fecal contamination (Commission Regulation, 2007). Some isolates are not pathogenic and are added to foods (such as cheeses) to extend their shelf life and improve their sensory properties (Giraffa, 2003). Some isolates of dairy origin also produce bacterocins (enterocins) that inhibit the growth of food spoilage or pathogenic bacteria, such as Listeria monocytogenes, Staphylococcus aureus, Vibrio cholerae, Clostridium spp., and Bacillus spp. (Giraffa, 2003; Cocolin et al., 2007).

Enterococcus faecalis is a member of the lactic acid bacteria (LAB) that consists of gram-positive cocci or rods that predominantly produce lactic acid as a by-product of carbohydrate fermentation. Unlike most LAB, E. faecalis cannot be considered as "generally recognized as safe" because some isolates are potential pathogens, especially in the nosocomial environment, and some of them were considered as a reservoir of genes encoding antibiotic resistance, which could be transferred to other microorganisms in the gut environment (Pesavento et al., 2014). So, understanding the relationship among the isolates from different sources is becoming more important.

In recent years, various genotyping methods were used to identify isolates or to further track their sources, including pulsed field gel electrophoresis, multiple variable number tandem repeat analysis, and multilocus sequence typing (MLST). Among them, MLST is a popular one. Nallapareddy et al. (2002) first evaluated the discriminatory power of MLST compared with pulsed field gel electrophoresis for E. faecalis and in his pilot study showed that sequence-based typing had potential to differentiate the isolates at the subspecies level and identify outbreak isolates. Subsequent studies confirmed the potential of MLST as an excellent tool for isolate characterization and long-term epidemiologic analysis in the related species E. faecium (Homan et al., 2002).

Although some studies have been done on the properties and epidemiological characteristics of E. faecalis, few studies are available on the relationship between 
Table 1. Isolates of Enterococcus faecalis and their sources

\begin{tabular}{|c|c|c|c|}
\hline Isolate reference number & \multicolumn{3}{|c|}{ Source } \\
\hline IMAU 10057, IMAU10119, IMAU10134 & Yogurt & $\begin{array}{l}\text { Wulatezhong Banner, Bayan Nur City, } \\
\text { Inner Mongolia }\end{array}$ & 2002 \\
\hline $\begin{array}{l}\text { IMAU10060, IMAU10063, IMAU10064, IMAU10075, } \\
\text { IMAU10078, IMAU10130, IMAU10133 }\end{array}$ & Fermented sheep milk & $\begin{array}{l}\text { Wulatezhong Banner, Bayan Nur City, } \\
\text { Inner Mongolia }\end{array}$ & 2002 \\
\hline IMAU10087 & Cow butter & $\begin{array}{l}\text { Wulatezhong Banner, Bayan Nur City, } \\
\text { Inner Mongolia }\end{array}$ & 2002 \\
\hline IMAU10095 & Sheep-milk cheese & $\begin{array}{l}\text { Wulatehou Banner, Bayan Nur City, Inner } \\
\text { Mongolia }\end{array}$ & 2002 \\
\hline IMAU10351, IMAU10440, IMAU10483 & Yogurt & $\begin{array}{l}\text { New Barag Left Banner, Hulunbeier } \\
\text { League, Inner Mongolia }\end{array}$ & 2009 \\
\hline IMAU10826, IMAU10861, IMAU10868, IMAU10917 & Yogurt & Balinyuo Banner, Chifeng, Inner Mongolia & 2009 \\
\hline IMAU10052 & Sheep butter & $\begin{array}{l}\text { Wulatezhong Banner, Bayan Nur City, } \\
\text { Inner Mongolia }\end{array}$ & 2002 \\
\hline IMAU40046 & Yak milk & $\begin{array}{l}\text { Gonghe County, Hainan Profecture, } \\
\text { Qinghai }\end{array}$ & 2005 \\
\hline IMAU60007 & Yogurt & Gyangze County, Shigatse area, Tibet & 2007 \\
\hline IMAU60129, IMAU60134, IMAU60135 & Yogurt & Danxung County, Lhasa area, Tibet & 2007 \\
\hline IMAU60196 & Yak milk & Gyangze County, Shigatse area, Tibet & 2007 \\
\hline C19315WT & Human pathogens & $\begin{array}{l}\text { Broad Institute of MIT and Harvard, } \\
\text { Cambridge, MA }\end{array}$ & 2013 \\
\hline $\mathrm{T} 2$ & Human urine & Sapporo, Japan & 2009 \\
\hline RP2S-4 & Nonhuman source & Unknown & 2007 \\
\hline V583 & $\begin{array}{l}\text { Blood culture derived } \\
\text { from a chronically } \\
\text { infected patient }\end{array}$ & Barnes Hospital, St. Louis, MO & 1987 \\
\hline
\end{tabular}

properties of E. faecalis, particularly whether they are pathogens, probiotics, or otherwise useful in the food industry, and their original source. Moreover, the related data for E. faecalis from food sources were limited. In this study, we tried to compare the genetic profile of the strains from Chinese traditional food products with some human pathogens, and then provide some more data for the MLST analysis of E. faecalis.

\section{MATERIALS AND METHODS}

\section{Bacterial Isolates and DNA Extraction}

A total of 39 E. faecalis isolates were obtained from the Collection Centre of Lactic Acid Bacteria of Inner Mongolia Agriculture University, China, and identified by $16 \mathrm{~S}$ rRNA analysis. These isolates originated from traditional Chinese food products (including yogurt, fermented sheep milk, cow butter, sheep-milk cheese, kurut, yak milk, acidic gruel, and sheep butter) from 10 regions in 3 provinces of China (Inner Mongolia, Qinghai, and Tibet) from 2002 to 2009 (Table 1). Stock cultures were stored in $10 \%$ glycerol at $-80^{\circ} \mathrm{C}$.
Working cultures were activated by 2 subcultures in M17 broth (Oxoid, Unipath Ltd., Basingstoke, UK), each at $37^{\circ} \mathrm{C}$ for 18 to $24 \mathrm{~h}$ under anaerobic conditions. The related sequences of another 4 reference isolates of E. faecalis (C19315WT, RP2S-4, T2, and V583) were obtained from the National Center for Biotechnology Information database (http://www.ncbi.nlm.nih.gov/ genome/?term=Enterococcus + faecalis). Enterococcus faecalis $\mathrm{RP} 2 \mathrm{~S}-4$ was isolated from an unknown nonhuman source, but the others were all human pathogens (Table 1).

Each isolate was cultured overnight at $37^{\circ} \mathrm{C}$ in $\mathrm{M} 17$ broth and then the total genomic DNA was extracted using the methods described by Dan et al. (2014).

\section{MLST Analysis}

Purified DNA was diluted to a final concentration of $100 \mathrm{ng} / \mu \mathrm{L}$ for evaluation. Primers were designed using Premier 5.0 (Table 2) based on the consensus sequences of 5 housekeeping genes in E. faecalis V583 ( pepC, clpX, rec $A, r p o B$, and groEL) and used to amplify fragments from those 5 housekeeping genes. General standards 
Table 2. Genes and primer sequences used

\begin{tabular}{|c|c|c|c|c|c|}
\hline Gene & Gene product & Primer $^{1}$ & Sequence $\left(5^{\prime} \rightarrow 3^{\prime}\right)$ & $\begin{array}{l}\text { Length } \\
\text { (bp) }\end{array}$ & $\begin{array}{c}\text { Annealing } \\
\text { temperature }\left({ }^{\circ} \mathrm{C}\right)\end{array}$ \\
\hline рер $C$ & Aminopeptidase $\mathrm{C}$ & $\begin{array}{l}\text { рер } C / \mathrm{F} \\
\operatorname{ep} C / \mathrm{R}\end{array}$ & $\begin{array}{l}\text { AGCAGCAACAGTTCCAAT } \\
\text { ATCCACGCATCGCTCATA }\end{array}$ & 725 & 51.1 \\
\hline groEL & Chaperonin GroEL & $\begin{array}{l}\text { groEL/F } \\
\operatorname{gro} E L / \mathrm{R}\end{array}$ & $\begin{array}{l}\text { GCGGATGATGTTGATGGG } \\
\text { AGCGATTTGACGGATTGG }\end{array}$ & 612 & 56.6 \\
\hline $\operatorname{clp} X$ & ATP-dependent protease subunit Clp X & $\begin{array}{l}\operatorname{clp} X / \mathrm{F} \\
\operatorname{clp} X / \mathrm{R}\end{array}$ & $\begin{array}{l}\text { GATGAAGCAGTCCGAGAA } \\
\text { TACAGGTAAGCGTCCAAT }\end{array}$ & 765 & 51.1 \\
\hline
\end{tabular}

${ }^{1} \mathrm{~F}=$ forward; $\mathrm{R}=$ reverse.

for selecting genes for the MLST analysis include even separation across the chromosome, functions of the encoded proteins, and the presence in all the strains as a single copy.

The PCR procedure consisted of an initial denaturizing step at $94^{\circ} \mathrm{C}$ for $4 \mathrm{~min} ; 30$ cycles of $94^{\circ} \mathrm{C}$ for 1 min, annealing at 50 to $60^{\circ} \mathrm{C}$ (optimal annealing temperatures for each locus were listed in Table 2) for $45 \mathrm{~s}$, and $72^{\circ} \mathrm{C}$ for $1 \mathrm{~min}$, and a final extension step at $72^{\circ} \mathrm{C}$ for $7 \mathrm{~min}$. The $50-\mu \mathrm{L}$ reaction mixtures contained 150 ng of genomic DNA, $10 \mathrm{~m} M$ of each dNTP, $10 \mathrm{pmol}$ of each primer, and $2.5 \mathrm{U}$ of Taq polymerase in $1 \times$ PCR buffer (with $\mathrm{Mg}^{2+}$ ). The PCR products were separated by electrophoresis in a $1.2 \%$ agarose gel and the visualized products sequenced by Shanghai Majorbio Bio-pharm Technology Corporation (Shanghai, China). The sequences were deposited in the GenBank library (http://www.ncbi.nlm.nih.gov/nuccore) under accession numbers KP134588-KP134782.

\section{Data Analysis}

The trimmed sequences obtained for the 5 housekeeping genes of all isolates in the MLST scheme were imported into BioNumerics Software (version 6.0, Applied-Maths, Saint Maartens-Latem, Belgium) to define alleles and construct a minimum spanning tree. All unique sequences were assigned an allele number and every unique/unambiguous combination of 5 allele numbers (the allelic profile) was designated as a sequence type (ST). When isolates shared the same allelic profiles, they were allocated to the same ST. The mean $\mathrm{G}+\mathrm{C}$ content, $d_{\mathrm{N}} / d_{\mathrm{S}}$ ratios (where $d_{\mathrm{S}}=$ the number of synonymous substitutions per synonymous site, and $d_{\mathrm{N}}=$ the number of nonsynonymous substitutions per nonsynonymous site), the number of polymorphic sites, and SNP in the 5 housekeeping genes for each isolate were calculated using START v2.0 (Jolley et al., 2001). The index of association $\left(\mathrm{I}_{\mathrm{A}}\right)$ [derived from the equation, $\mathrm{I}_{\mathrm{A}}=\mathrm{V}_{\mathrm{O}} / \mathrm{V}_{\mathrm{E}}-1\left(\mathrm{~V}_{\mathrm{O}}=\right.$ observed variance; $\mathrm{V}_{\mathrm{E}}=$ expected variance)] and the standardized index of association $\left(\mathrm{I}_{\mathrm{A}}{ }^{\mathrm{S}}\right.$; calculated by LIAN 3.0) were used to evaluate the level of linkage disequilibrium between all alleles (Haubold and Hudson, 2000; Suerbaum et al., 2001). Nucleotide diversity $(\pi)$ per site was calculated using DnaSP version 5.0 (Rozas et al., 2003). The groups of isolates with the most closely related allelic profiles were placed within clonal complexes $(\mathbf{C C})$. The relationships between ST and the analysis of CC were made using the eBURST program located within the BioNumerics program (Feil et al., 2004). To evaluate the potential influence of recombination on evolutionary dynamics, the degree of tree-like structures for alleles of each locus and all ST were assessed with the split decomposition method using SplitsTree 4.0 (Huson and Bryant, 2006). A neighbor-joining tree was constructed by Mega 5.0 software (www.megasoftware.net).

\section{RESULTS}

\section{MLST Scheme and Allelic Variation}

To identify each locus accurately, we used bidirectional sequence methods. For all isolates, the 5 genes were successfully sequenced and analyzed by MLST. Polymorphic sites, GC content, rate of nonsynonymous $\left(d_{\mathrm{N}}\right)$, synonymous $\left(d_{\mathrm{S}}\right)$ substitutions, and the $d_{\mathrm{N}} / d_{\mathrm{S}}$ ratio for each locus ( were determined (Table 3 ). The mean GC content of the partial sequences of the 5 gene fragments ranged from $34.69 \%$ ( lar with the $37.50 \%$ value observed across the entire $E$. faecalis V583 genome (Shankar et al., 2002; Yu et al., 2014). Trimmed fragment sizes of the 5 selected loci ranged from $479 \mathrm{bp}($ groEL $)$ to $619 \mathrm{bp}(\operatorname{clp} X)$. The average nucleotide diversity $\pi$ was 0.03258 , ranging from 0.00427 to 0.07598 per gene. Moreover, the number of polymorphic sites per locus ranged from $8(p e p C, \operatorname{clp} X)$ 
Table 3. Nucleotide and allelic diversity of the 5 housekeeping genes evaluated

\begin{tabular}{|c|c|c|c|c|c|c|}
\hline Locus & $\begin{array}{l}\text { Size } \\
\text { (bp) }\end{array}$ & Alleles & $\begin{array}{l}\text { Polymorphic } \\
\text { sites }\end{array}$ & $\begin{array}{c}\text { GC content } \\
(\%)\end{array}$ & $\pi^{1}$ & $d_{\mathrm{N}} / d_{\mathrm{S}}^{2}$ \\
\hline рерC & 572 & 9 & 8 & 34.69 & 0.00427 & 0.0265 \\
\hline $\operatorname{clp} X$ & 619 & 10 & 8 & 37.11 & 0.00434 & 0.0000 \\
\hline recA & 557 & 8 & 99 & 43.38 & 0.07598 & 0.0174 \\
\hline rро $B$ & 565 & 10 & 110 & 39.89 & 0.07021 & 0.0667 \\
\hline groEL & 479 & 9 & 12 & 40.32 & 0.00812 & 0.0000 \\
\hline
\end{tabular}

to $110($ rpo $B)$ and harbored a total of 237 SNP. The $d_{\mathrm{N}} /$ $d_{\mathrm{S}}$ ratios for the 5 loci were all less than 1 (2 genes $=$ 0 ), indicating that these housekeeping genes had undergone negative selection.

\section{Assignment of Sequence Type}

Forty-three E. faecalis isolates were typed using the MLST protocol. Isolates could be divided into $23 \mathrm{ST}$ using combined data from 5 loci (Table 4). The ST5 was the most frequent sequence type and included 7 isolates of E. faecalis; they were all from animal milk or related products, including yogurt (2), fermented sheep milk (2), kurut (2), and yak milk (1). The next most frequent ST was ST6, which included 6 isolates of E. faecalis from traditional dairy products [fermented sheep milk (3), butter (1), and yogurt (2)] in Inner Mongolia. The ST2, ST7, and ST19 each included 3 isolates. The 3 isolates in ST2 were from fermented dairy products [yogurt (1), fermented sheep milk (2)] from the same place. The 3 isolates in ST7 were from different traditional dairy products in Inner Mongolia, specifically 2 from fermented sheep milk, and one from yogurt. The isolates in ST19 were all from acidic gruel from Huhhot, Inner Mongolia. The ST4, ST8, and ST15 each included 2 isolates. The 2 isolates in ST4 were both from fermented sheep milk from Wulatezhong (Bayan Nur City, Inner Mongolia). Although the sources for the 2 isolates in ST8 were different, we can see that the product from which these 2 isolates originated was the same; they were both from sheep milk. In contrast, the 2 isolates in ST15 were from yogurts of different regions of Tibet. The other 15 ST only contained one isolate each. The ST20, ST21, ST22, and ST23 represented the 4 isolates from public database, respectively.

Table 4. Allelic profiles based on 5 housekeeping genes from all isolates of Enterococcus faecalis evaluated

\begin{tabular}{lccccll}
$\begin{array}{l}\text { Sequence } \\
\text { type }\end{array}$ & pepC & clp $X$ & recA & rpo $B$ & groEL & Isolate reference number \\
\hline 1 & 5 & 5 & 7 & 1 & 7 & IMAU10052 \\
2 & 6 & 2 & 1 & 3 & 6 & IMAU10057, IMAU10060, IMAU10063 \\
3 & 5 & 4 & 7 & 1 & 6 & IMAU10064 \\
4 & 7 & 8 & 1 & 2 & 2 & IMAU10075, IMAU10130 \\
5 & 5 & 4 & 2 & 2 & 7 & IMAU10078, IMAU10119, IMAU10133, IMAU10134, IMAU40025, IMAU40046, \\
& & & & & & IMAU40105 \\
6 & 3 & 7 & 1 & 1 & 7 & IMAU10087, IMAU10094, IMAU10098, IMAU10099, IMAU10861, IMAU10868 \\
7 & 2 & 1 & 3 & 1 & 1 & IMAU10091, IMAU10100, IMAU10440 \\
8 & 1 & 6 & 5 & 5 & 5 & IMAU10095, IMAU10103 \\
9 & 4 & 1 & 3 & 1 & 1 & IMAU10102 \\
10 & 8 & 5 & 6 & 7 & 4 & IMAU10351 \\
11 & 3 & 4 & 3 & 6 & 1 & IMAU10483 \\
12 & 2 & 4 & 3 & 6 & 1 & IMAU10826 \\
13 & 5 & 5 & 8 & 1 & 7 & IMAU10917 \\
14 & 5 & 7 & 2 & 2 & 1 & IMAU40027 \\
15 & 5 & 5 & 1 & 8 & 7 & IMAU60007, IMAU60134 \\
16 & 5 & 4 & 1 & 9 & 7 & IMAU60129 \\
17 & 5 & 4 & 1 & 2 & 7 & IMAU60135 \\
18 & 5 & 5 & 1 & 1 & 7 & IMAU60196 \\
19 & 6 & 3 & 4 & 4 & 3 & IMAU70078, IMAU70121, IMAU70122 \\
20 & 5 & 9 & 4 & 1 & 8 & Es.C19315WT \\
21 & 5 & 9 & 1 & 1 & 2 & Es.RP2S-4 \\
22 & 6 & 10 & 4 & 4 & 3 & Es.T2 \\
23 & 9 & 5 & 4 & 10 & 9 & Es.V583 \\
\hline
\end{tabular}




\section{Recombination in E. faecalis}

The level of linkage disequilibrium between all alleles of the isolates was evaluated by calculating the index of association $\mathrm{I}_{\mathrm{A}}$ and standardized index of association $\mathrm{I}_{\mathrm{A}}{ }^{\mathrm{S}}$. Calculated values for $\mathrm{I}_{\mathrm{A}}$ and $\mathrm{I}_{\mathrm{A}}{ }^{\mathrm{S}}$ were 0.5862 and $0.1465(P=0.000)$, respectively, demonstrating that these 5 loci expressed significant linkage disequilibrium. In other words, clonal structures were present among this set of E. faecalis isolates.

Split decomposition analysis was used to examine the evolutionary relationships among all the isolates (Figure 1a). This analysis showed parallelogram-shaped structures for $\operatorname{clp} X, g r o E L$, and $p e p C$, indicating that these genes had undergone intergenic recombination during their evolution. For the remaining 2 loci, the split graphs revealed a tree-like structure, suggesting that the descent of these genes was clonal and not significantly affected by intergenic recombination. Combined split-graph analysis of alleles for all 5 MLST loci showed that the 23 ST representing all isolates were divided into 3 main subpopulations and each subpopulation was completely disconnected (Figure 1b). The ST1, ST3, and ST13 were clustered together as one subpopulation. The 3 isolates in this subpopulation were all from the traditional dairy products of Inner Mongolia. The ST15 and ST16 were clustered together as the second subpopulation. The 3 isolates in this subpopulation were all from the traditional fermented yogurt in Tibet. The remaining ST were clustered together as the third subpopulation, but no relationships seemed to be present between the source and sampling regions of these isolates.

\section{Neighbor-Joining Tree Based on MLST Data}

Phylogenetic relatedness among the E. faecalis ST investigated in this study was evaluated and represented as a neighbor-joining tree based on the concatenated sequences of 5 loci (Figure 2).

All the isolates clustered into 3 major groups. This classification was in accordance with that described above. Specifically, group B was composed of ST1, ST3, and ST13; the 3 isolates in this group were all from traditional dairy products in Inner Mongolia. Group C contained ST15 and ST16, the isolates in this group were all from the homemade yogurt in Tibet. The left 18 ST composed group A, including the 4 type strains. In this group, particular pairs of ST were clearly closely related, specifically ST19 and ST22, ST7 and ST9, and ST11 and ST12. Except ST19 and ST22, the other pairs were all from similar sources. The ST19 included the 3 isolates from acidic gruel. However, ST22 represented the type strain E. faecalis T2, which was from urine and is considered a human pathogen.

\section{Cluster Analysis of the MLST Data}

Clustering by source was evident among the isolates in the minimum-spanning tree (Figure 3). The $43 \mathrm{E}$. faecalis isolates evaluated were assigned to $23 \mathrm{ST}$ that resolved into 2 major CC and 3 minor CC. Ten singleton ST that could not be assigned to any group were also apparent.

The largest CC1 consisted of ST1, ST15, ST18, and ST13, which included 5 isolates, mainly from the traditional dairy products of Inner Mongolia and Tibet. Within this CC, ST18 was the predicted primary founder surrounded by a single locus (ST1, ST13, ST15). These ST were closely related as expressed by solid black lines. The CC2 comprised ST5, ST16, and ST17, which included 9 isolates mainly from the traditional dairy products of Inner Mongolia and Tibet. Within this CC, ST 17 was the predicted primary founder surrounded by a single locus (ST5, ST16). The CC3 was composed of ST7 and ST9, which included 4 isolates from fermented milk products from Inner Mongolia. The CC4 was composed of ST11 and ST12, which included 2 isolates from yogurt samples from Inner Mongolia. The CC5 was composed of ST19 and ST22. Within this CC, the type isolate $E$. faecalis $\mathrm{T} 2$ had a close relationship with the 3 isolates from acidic gruel in Inner Mongolia, which was in accordance with the results described previously. The ST2, ST3, ST4, ST6, ST8, ST10, ST14, ST20, ST21, and ST23 were singletons unlinked to the other CC. However, they were all connected to the 2 founders, by gray or dotted lines, indicating a distant relationship with these 2 predicted ancestors. The ST6 and ST21 were 2-locus variants of ST18; and ST3 and ST20 were 3-locus variants of ST18. The ST4 and ST14 were 3-locus variants of ST17. The ST2, ST10, and ST23 were 4-locus variants of ST18, respectively. The ST8 was a 5-locus variant of ST18.

\section{DISCUSSION}

Enterococcus species are ubiquitous bacteria that frequently occur in large numbers in dairy and other food products. Some isolates show several beneficial biotechnological properties such as bacteriocin production, probiotic characteristics, and usefulness in dairy technology; however, other isolates can cause human infections. It is, therefore, necessary to determine the relationships among $E$. faecalis isolates from different sources to aid in evaluating their possible safe use in industrial applications. Most previous studies showed 
(a)
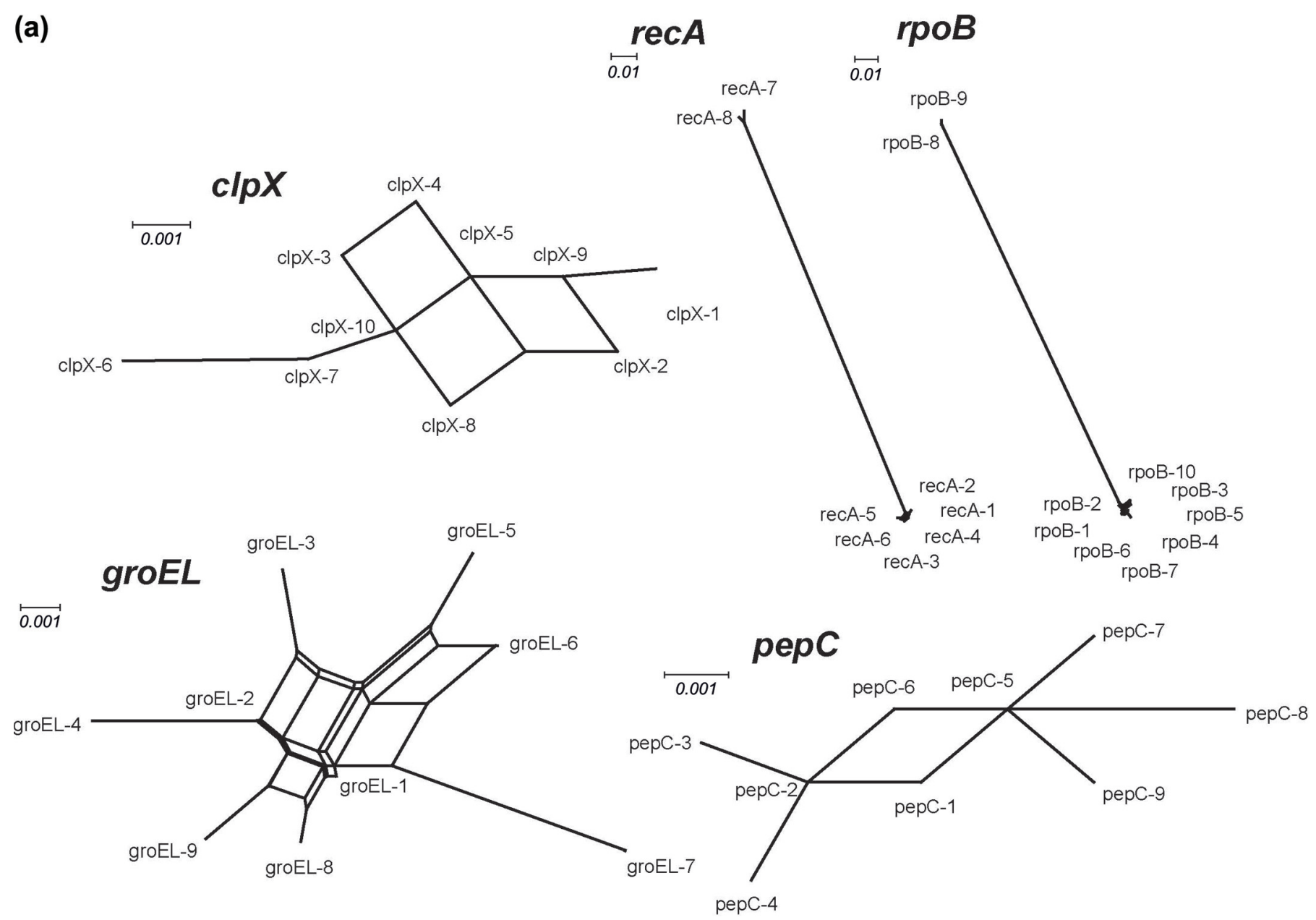

Figure 1. Split-decomposition analysis based on concatenated sequences of 5 housekeeping genes from 43 Enterococcus faecalis isolates. Note: multiparallelogram formations indicate recombination events. (a) Split-decomposition analysis of individuals multilocus sequence typing (MLST) loci; (b) combined split decomposition analysis of all 5 MLST loci.

that MLST was useful to accurately identify bacterial lineages (Urwin and Maiden, 2003), but few studies have considered the relationships between isolates and their source, let alone the isolates from Chinese traditional food products. In this study, we used MLST to type E. faecalis isolates from different food-related sources and look for relatedness to isolates that were human pathogens. These representative isolates were unique in their diversity of sources and provide some necessary information required to better understand genetic diversity, persistence, and movement in this species.

To ensure the MLST protocol had sufficient discriminatory power to type isolates within a single species, it was necessary to first analyze sequence diversity of 5 housekeeping genes from the 43 E. faecalis isolates. Three loci had low polymorphism ( $p e p C, \operatorname{clpX}$, and gro$E L$ loci), indicated that they had similar sequences at the species level and might, therefore, offer a lower discriminatory ability compared with other housekeeping loci (Dan et al., 2014). The remaining 2 loci, recA and rрo $B$ had 99 and 102 sites, respectively, suggesting recombination was evident and representing a significant source of genetic diversity in E. faecalis. High diversity in rec $A$ alleles and evidence for frequent recombination at this locus has been observed in previous studies (Han et al., 2014). In general, the allelic diversity of all 5 genes was less than 25\%, indicating that the MLST design was balanced and corresponded with accepted designation principles (Maiden, 2006). According to our analysis of E. faecalis using MLST, these 2 loci were the most influential on apparent phylogenetic relationships and population structure of E. faecalis. During the genetic analysis, the 5 housekeeping loci all had a very low $d_{\mathrm{N}} / d_{\mathrm{S}}$ ratio $(<1)$, which indicated negative selection. For $c l p X$ and groEL, the $d_{\mathrm{N}} / d_{\mathrm{S}}$ ratios were close to zero, so we can conclude that they were synonymous and the amino acid composition did not change. These results are consistent with the findings 

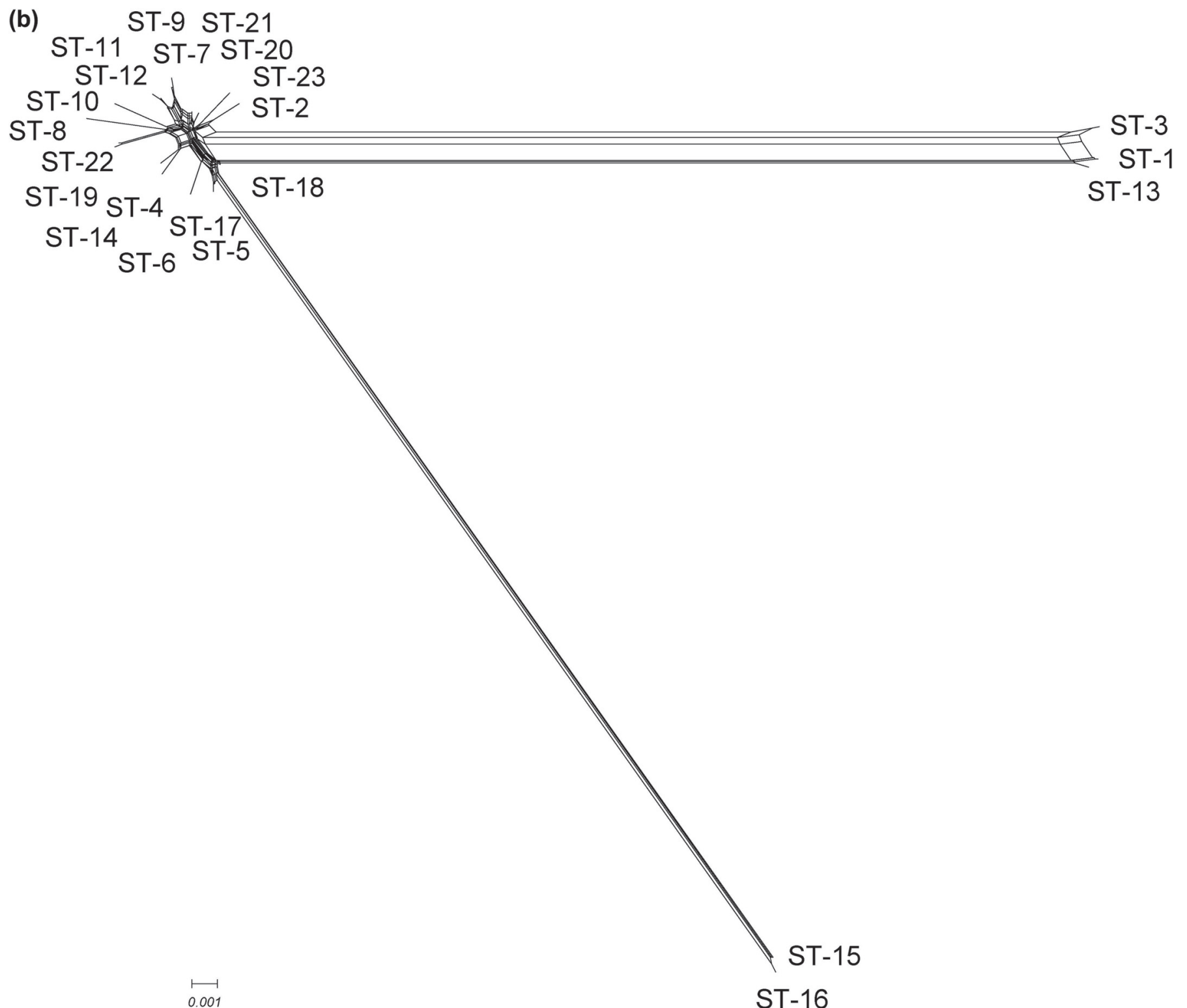

Figure 1 (Continued). Split-decomposition analysis based on concatenated sequences of 5 housekeeping genes from 43 Enterococcus faecalis isolates. Note: multiparallelogram formations indicate recombination events. (a) Split-decomposition analysis of individuals multilocus sequence typing (MLST) loci; (b) combined split decomposition analysis of all 5 MLST loci.

of Sun et al. (2015), and they support our belief that the housekeeping loci are all under negative selection.

The $\mathrm{I}_{\mathrm{A}}$ is a generalized measure of linkage disequilibrium and has no relationship with the number of loci analyzed. If no association among the loci is checked, the value will be zero, which means alleles are in linkage equilibrium (free recombination; Suerbaum et al., 2001). In our study, the values of $\mathrm{I}_{\mathrm{A}}$ and $\mathrm{I}_{\mathrm{A}}{ }^{\mathrm{S}}$ for 5 loci were calculated as 0.5862 and $0.1465(P=0.000)$, respectively. These values are indicative of a clonal population, and suggested that allelic selection was close to linkage disequilibrium. Besides $\mathrm{I}_{\mathrm{A}}^{\mathrm{S}}$, the number of unique ST reflects the genetic diversity among isolates that have each adapted to specialized environments during their evolution.

Many previous studies have shown that Enterococcus species express high genetic diversity. For example, 13 ST with different allelic profiles were identified from the polymorphic regions of 4 genes (ace, efaA, pyrC, and salA) from $22 \mathrm{E}$. faecalis isolates (Nallapareddy et al., 2002). A further study derived 62 ST by analyzing the internal fragments from 7 housekeeping genes $(g d h$, 


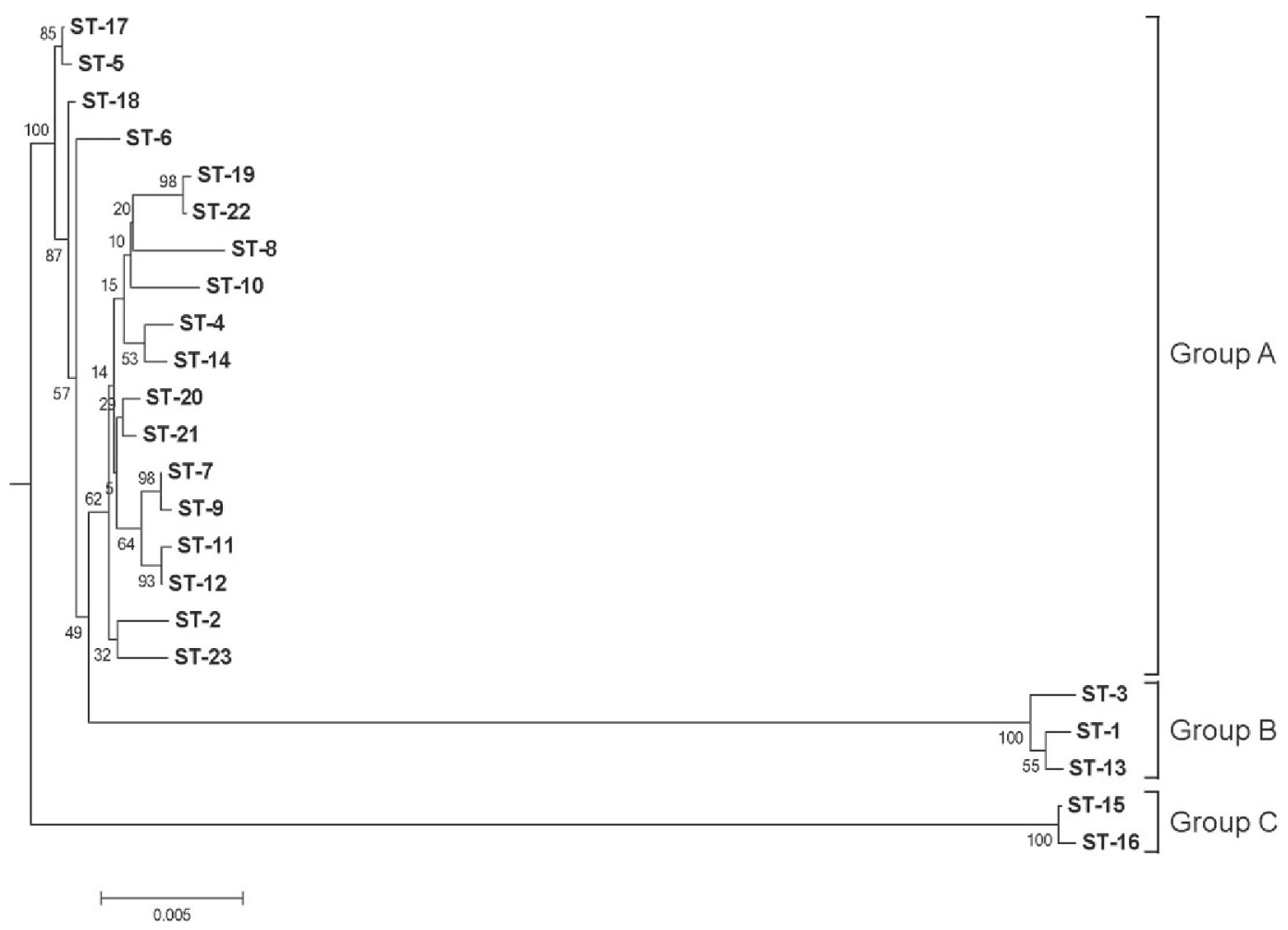

Figure 2. Neighbor-joining tree generated based on the concatenated sequences of five loci in the Enterococcus faecalis isolates evaluated.

purK, pstS, atpA, gyd, adk, and $d d l)$ from 123 epidemiologically unlinked human and livestock-derived isolates and 16 human-derived isolates of E. faecium from several outbreaks in the United States, the United Kingdom, Australia, and the Netherlands (vancomycin-sensitive and vancomycin-resistant isolates; Homan et al., 2002). Subsequently, Bonora et al. (2004) identified 9 ST from 14 vancomycin-resistant E. faecium isolates by MLST based on 7 housekeeping genes ( $a d k, a t p A, d d l$, gyd, gdh, purK, and pstS), and Camargo et al. (2006) identified 26 ST within 3 groups with related allelic profiles from 56 vancomycin-resistant and vancomycinsensitive E. faecium isolates. Our study was in line with these previous studies as we detected 23 ST from 43 isolates of E. faecalis. In our study, ST5 occurred most frequently and was represented by isolates from milkrelated sources, such as fermented sheep milk, yogurt, kurut, and yak milk. A similar association was found for isolates in the second most frequent sequence type, ST6, which were derived from butter, fermented sheep milk, and cow milk. The results suggested the source of the isolate might influence its sequence type.

Split decomposition analysis based on the allelic profiles of isolates has provided evidence of intraspecies recombination that could play a role in generating genotypic diversity among isolates. In our study, parallelogram-shaped structures or treelike structures were commonly found in the split graphs for all the 5 housekeeping genes evaluated, illustrating that recombination had occurred in these MLST loci (Figure 1a). This result was similar to that of previous studies. Ruiz-Garbajosa et al. (2006) provided evidence that recombination was an important mechanism driving genetic variation in the E. faecalis isolates they evaluated.

Although our study has provided important insight into the population structure of E. faecalis, particularly from the split-decomposition analysis based on concatenated sequences of housekeeping genes (Figure 1b) and the neighbor-joining tree (Figure 2) based on the MLST data, we could still not confirm categorically any association between ST and the original source of each isolate. This is similar to previous studies on other LAB species including Leuconostoc lactis (Dan et al., 2014), Lactococcus lactis (Passerini et al., 2010), and Lactobacillus sanfranciscensis (Picozzi et al., 2010). The absence of such an association in E. faecalis might be caused by the genetic diversity within individual $E$. faecalis isolates.

Interestingly, the MLST analysis showed that ST22 and ST19 were clustered together (Figure 3). The ST19 comprised 3 isolates from traditional acidic gruel, but ST22 was represented by E. faecalis isolate T2, which 


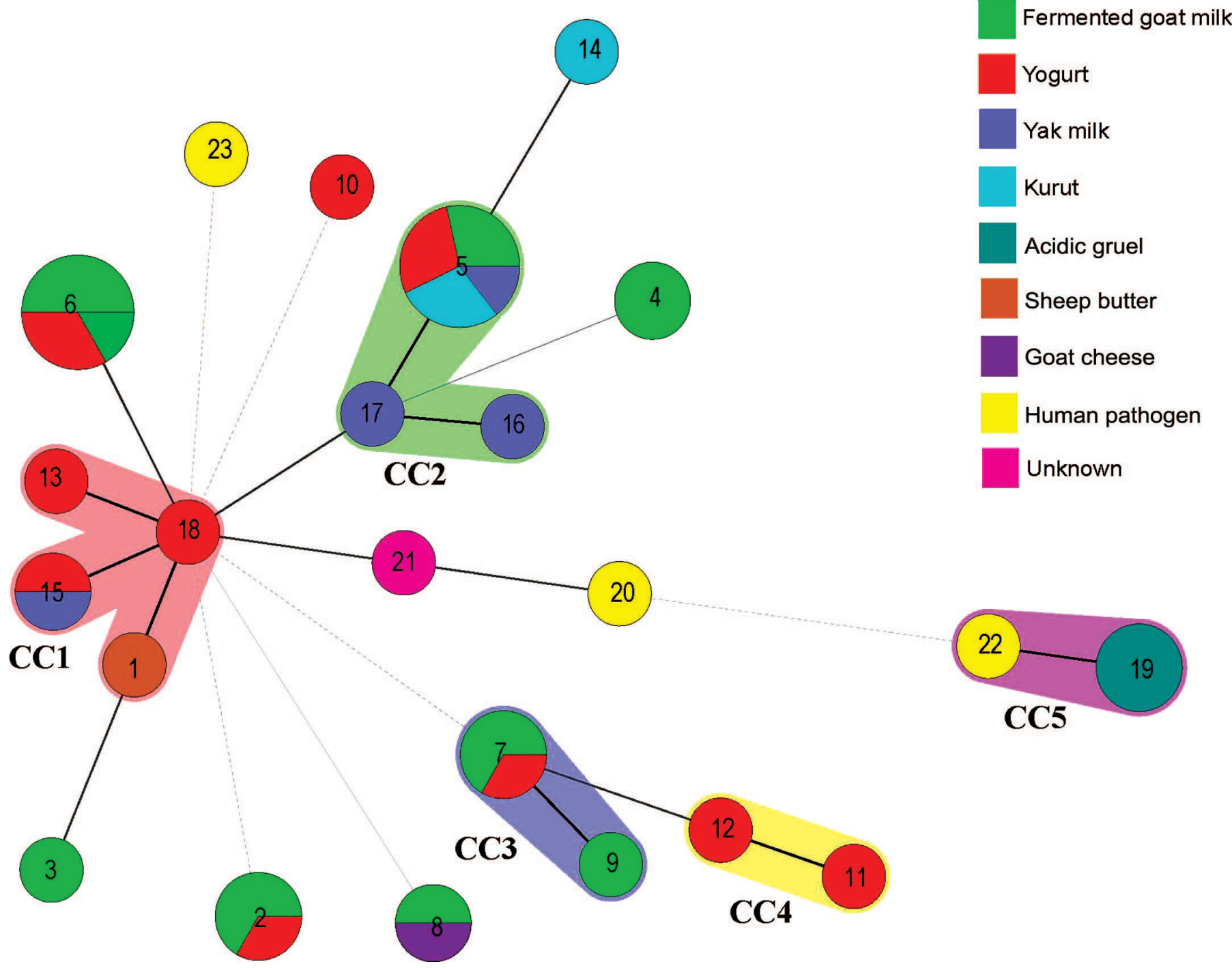

Figure 3. Minimum-spanning tree analyses of 43 Enterococcus faecalis isolates based on multilocus sequence typing (MLST) data according to source. Note: each circle indicates a sequence type (ST), different number means different ST. The size of the circle is proportional to the number of isolates within it. The shaded zones between particular groups of circles indicate that these profiles belong to the same clonal complex (CC). The type of line between isolates indicates the strength of the link (black line means intermediate relationship, dotted line means weak relationship) of genetic similarity between profiles.

is a human pathogen isolated from urine in Sapporo, Japan, and obtained from the National Center for Biotechnology Information database. Acidic gruel (also known as sour congee) is a traditional, fermented cereal gruel that has been consumed for hundreds of years in the western regions of Inner Mongolia. The production procedure of acidic gruel was as follows: cereal grains, mainly millet and rice (sometimes blended with a little maize), are soaked with clean water in a pot at room temperature (about $25^{\circ} \mathrm{C}$ ) for 3 to $4 \mathrm{~d}$. During fermentation, mixed fermentations including lactic acid fermentation take place, and the $\mathrm{pH}$ decreases as lactic acid and other organic acids accumulate due to micro- bial activities. Subsequently, a part of the fermented product is added into the new soaked rice mixture as a starter for fermentation at room temperature until the mixture produces acid, and then the fermented mixture is boiled, and the palatable and nutritional acidic gruel is ready for direct consumption ( $\mathrm{Yu}$ et al., 2011). Acidic gruel is passed on from one generation to another, so it preserves the specific microflora of the local environment. Therefore, it is unsurprising that the relationship between the isolates from acidic gruel and human source was closely related, based on the MLST analysis. We will try to isolate more E. faecalis strains from acidic gruel for further analysis, and more rigorous 
safety evaluations might be needed when considering the industrial application of the isolates derived from acidic gruel was suggested. The isolate of E. faecalis RP 2S-4, from the National Center for Biotechnology Information database, was described as "isolated from nonhuman sources, provided by Donna Ferguson and Blythe Layton (SCCWRP, Southern California Coastal Water Research Project, 3535 Harbor Blvd., Suite 110 Costa Mesa, CA 92626)." So, it is, perhaps, not surprising that this reference isolate did not show a relatively close relationship with ST20 and ST23. The ST20 represents E. faecalis C19315WT, which was also a human pathogen isolated from human-derived samples held at the Broad Institute of MIT and Harvard, 7 Cambridge Center, Cambridge, MA 02142. The E. faecalis isolate V583 represented by ST23 was one of the first vancomycin-resistant isolates from blood (derived from a chronically infected patient in 1987 from Barnes Hospital in St. Louis, Missouri) and lacked the cytolysin gene and a surface adhesin, which contribute to urinary tract infection (Paulsen et al., 2003; Wecker et al., 2009). From MLST analysis, we can clearly see a distant relationship between the isolates from traditional dairy products and these 2 human pathogens.

\section{CONCLUSIONS}

An MLST protocol for E. faecalis was developed based on 5 housekeeping genes and $43 \mathrm{E}$. faecalis isolates from different sources. The genetic biodiversity, clonal population structure, and genetic recombination among the selected isolates were evaluated. Five CC were obtained from the $23 \mathrm{ST}$ identified. The results also showed that the relationship between isolates from acidic gruel and one isolate from a human source was close; this might suggest that more rigorous safety evaluations are necessary when considering industrial applications of isolates from traditional acidic gruel in food production. In the future, we will choose more E. faecalis isolates from a wider range of human and nonhuman sources (especially acidic gruel) and examine their relationship by MLST protocol to further elucidate the evolution and population genetics of E. faecalis.

\section{ACKNOWLEDGMENTS}

This work was supported by Hi-Tech Research and Development Program of China [863 Planning, grant number 2011AA100901, 2011AA100902], International S\&T Cooperation Program of China [ISTCP, grant number 2014DFR31150], and the Special Fund for Agro-scientific Research in the Public Interest [grant number 201303085].

\section{REFERENCES}

Bonora, M. G., M. Ligozzi, M. De Fatima, L. Bragagnolo, A. Goglio, G. C. Guazzotti, and R. Fontana. 2004. Vancomycin-resistant Enterococcus faecium isolates causing hospital outbreaks in northern Italy belong to the multilocus sequence typing $\mathrm{C} 1$ lineage. Microb. Drug Resist. 10:114-123.

Cai, H., B. T. Rodríguez, W. Zhang, J. R. Broadbent, and J. L. Steele. 2007. Genotypic and phenotypic characterization of Lactobacillus casei strains isolated from different ecological niches suggests frequent recombination and niche specificity. Microbiology 153:2655-2665.

Camargo, I. L., M. S. Gilmore, and A. L. Darini. 2006. Multilocus sequence typing and analysis of putative virulence factors in vancomycin-resistant and vancomycin-sensitive Enterococcus faecium isolates from Brazil. Clin. Microbiol. Infect. 12:1123-1130.

Cocolin, L., R. Foschino, G. Comi, and M. Grazia Fortina. 2007. Description of the bacteriocins produced by two strains of Enterococcus faecium isolated from Italian goat milk. Food Microbiol. 24:752-758.

Commission Regulation. 2007. Commission Regulation (EC) No 1441/2007 of 5 December 2007 amending Regulation (EC) No 2073/2005 on microbiological criteria for foodstuffs. Official Journal of the European Union.

Dan, T., W. Liu, Z. Sun, Q. Lv, H. Xu, Y. Song, and H. Zhang. 2014. A novel multi-locus sequencing typing (MLST) protocol for Leuconostoc lactis isolates from traditional dairy products in China and Mongolia. BMC Microbiol. 14:150.

Feil, E. J., B. C. Li, D. M. Aanensen, W. P. Hanage, and B. G. Spratt. 2004. eBURST: Inferring patterns of evolutionary descent among clusters of related bacterial genotypes from multilocus sequence typing data. J. Bacteriol. 186:1518-1530.

Giraffa, G. 2003. Functionality of enterococci in dairy products. Int. J. Food Microbiol. 88:215-222.

Han, D., H. Tang, J. Lu, G. Wang, L. Zhou, L. Min, and C. Han. 2014 Population structure of clinical Vibrio parahaemolyticus from 17 coastal countries, determined through multilocus sequence analysis. PLoS ONE 9:e107371.

Haubold, B., and R. R. Hudson. 2000. LIAN 3.0: Detecting linkage disequilibrium in multilocus data. Bioinformatics 16:847-848.

Homan, W. L., D. Tribe, S. Poznanski, M. Li, G. Hogg, E. Spalburg, J. D. Van Embden, and R. J. Willems. 2002. Multilocus sequence typing scheme for Enterococcus faecium. J. Clin. Microbiol. 40:1963-1971.

Huson, D. H., and D. Bryant. 2006. Application of phylogenetic networks in evolutionary studies. Mol. Biol. Evol. 23:254-267.

Jolley, K. A., E. J. Feil, M. S. Chan, and M. C. Maiden. 2001. Sequence type analysis and recombinational tests (START). Bioinformatics 17:1230-1231.

Maiden, M. C. 2006. Multilocus sequence typing of bacteria. Annu. Rev. Microbiol. 60:561-588.

Nallapareddy, S. R., R. W. Duh, K. V. Singh, and B. E. Murray. 2002. Molecular typing of selected Enterococcus faecalis isolates: Pilot study using multilocus sequence typing and pulsed-field gel electrophoresis. J. Clin. Microbiol. 40:868-876.

Passerini, D., C. Beltramo, M. Coddeville, Y. Quentin, P. Ritzenthaler, M. Daveran-Mingot, and P. Le Bourgeois. 2010. Gene but not genomes reveal bacterial domestication of Lactococcus lactis. PLOS ONE 5:e15306.

Paulsen, I. T., L. Banejei, G. S. Myers, K. E. Nelson, R. Seshadri, T. D. Read, D. E. Fouts, J. A. Eisen, S. R. Gill, J. F. Heidelberg, H. Tettelin, R. J. Dodson, L. Umayam, L. Brinkac, M. Beanan, S. Daugherty, R. T. DeBoy, S. Durkin, J. Kolonay, R. Madupu, W. Nelson, J. Vamathevan, B. Tran, J. Upton, T. Hansen, J. Shetty, H. Khouri, T. Utterback, D. Radune, K. A. Ketchum, B. A. Dougherty, and C. M. Fraser. 2003. Role of mobile DNA in the evolution of vancomycin-resistant Enterococcus faecalis. Science 299:2071-2074.

Pesavento, G., C. Calonico, B. Ducci, A. Magnanini, and A. Lo Nostro. 2014. Prevalence and antibiotic resistance of Enterococcus 
spp. isolated from retail cheese, ready-to-eat salads, ham, and raw meat. Food Microbiol. 41:1-7.

Picozzi, C., G. Bonacina, I. Vigentini, and R. Foschino. 2010. Genetic diversity in Italian Lactobacillus sanfranciscensis strains assessed by multilocus sequence typing and pulsed-field gel electrophoresis analyses. Microbiology 156:2035-2045.

Rozas, J., J. C. Sánchez-DelBarrio, X. Messeguer, and R. Rozas. 2003. DnaSP, DNA polymorphism analyses by the coalescent and other methods. Bioinformatics 19:2496-2497.

Ruiz-Garbajosa, P., M. J. Bonten, D. A. Robinson, J. Top, S. R. Nallapareddy, C. Torres, T. M. Coque, R. Cantón, F. Baquero, B. E. Murray, R. del Campo, and R. J. Wilems. 2006. Multilocus sequence typing sheme for Enterococcus faecalis reveals hospitaladapted genetic complexes in a background of high rates of recombination. J. Clin. Microbiol. 44:2220-2228.

Shankar, N., A. S. Baghdayan, and M. S. Gilmore. 2002. Modulation of virulence within a pathogenicity island in vancomycin-resistant Enterococcus faecalis. Nature 417:746-750.

Suerbaum, S., M. Lohrengel, A. Sonnevend, F. Ruberg, and M. Kist. 2001. Allelic diversity and recombination in Campylobacter jejuni. J. Bacteriol. 183:2553-2559.
Sun, Z., W. Liu, Y. Song, H. Xu, J. Yu, M. Bilige, H. Zhang, and Y. Chen. 2015. Population structure of Lactobacillus helveticus isolates from naturally fermented dairy products based on multilocus sequence typing. J. Dairy Sci. 98:2962-2972.

Urwin, R., and M. C. Maiden. 2003. Multi-locus sequence typing: A tool for global epidemiology. Trends Microbiol. 11:479-487.

Wecker, P., C. Klockow, A. Ellrott, C. Quast, P. Langhammer, J. Harder, and F. O. Glöckner. 2009. Transcriptional response of the model planctomycete Rhodopirellula baltica $\mathrm{SH}^{\mathrm{T}}$ to changing environmental conditions. BMC Genomics 10:410.

Yu, J., X. Du, W. Wang, J. Zhang, W. Liu, Z. Sun, T. Sun, and H. Zhang. 2011. Phenotypic and genotypic characteristics of lactic acid bacteria isolated from sour congee in Inner Mongolia. J. Gen. Appl. Microbiol. 57:197-206.

Yu, Z., Z. Chen, H. Cheng, J. Zheng, D. Li, X. Deng, W. Pan, W. Yang, and Q. Deng. 2014. Complete genome sequencing and comparative analysis of the linezolid-resistant Enterococcus faecalis strain DENG1. Arch. Microbiol. 196:513-516. 